\title{
Nonlinear Evolution of Phase-mixed Alfvén Waves Towards Short Length Scales
}

\author{
M. Goossens ${ }^{1}$ and Y. Voitenko ${ }^{1,2}$ \\ ${ }^{1}$ Centre for Plasma Astrophysics, K.U.Leuven, Celestijnenlaan 200B, \\ 3001 Heverlee, Belgium \\ ${ }^{2}$ Main Astronomical Observatory, Golosiiv, 03680 Kyiv-127, Ukraine
}

\begin{abstract}
We study two nonlinear processes that are efficient in the spectral redistribution of Alfvén wave energy - second harmonic generation, and scalar decay. Both processes transport the energy of AWs towards smaller scales and/or higher frequencies, and can accelerate the energization of space plasmas implied by recent $\mathrm{SOHO}$ observations.
\end{abstract}

\section{Introduction}

Processes that transport energy to dissipative length-scales attract great interest in the space plasma physics community. Resonant absorption and phase mixing of Alfvén waves (see Goossens 1994, and references therein) are two popular linear mechanisms for transferring energy towards small length-scales. As the perpendicular wavenumber increases, the AWs begin to interact effectively with the plasma and accelerate plasma particles. Classic AWs undergo phase mixing in inhomogeneous space plasmas, but phase mixing on its own appears not to be able to create significant dissipative effects during required times.

Nonlinear interaction and turbulent cascade of AW energy to dissipative scales have been widely discussed recently in the context of space plasma energization ( Nakariakov, Roberts, \& Murawski 1997; Matthaeus et al. 1999; Li et al. 1999). However, as was mentioned by Matthaeus et al. (1999), the nonlinear dynamics of AWs, introduced by their anisotropic nature, is insufficiently explored.

Here we study two nonlinear processes that can strongly accelerate the evolution of AW energy towards small length scales: (i) parametric excitation of small-scale AWs by a large-scale pump AW due to scalar nonlinearity (non-local transfer in perpendicular wavenumber), and (ii) generation of AW at the second harmonic (local process in $\omega$ and $\mathbf{k}$ ).

\section{Mathematical model and eigenmode equation}

After a long calculation of which the details are given elsewhere (Voitenko \& Goossens 2000), we arrive at the following basic equation for AW and slow 
waves in terms of the effective density potential $\phi=(T / e) \ln \left(n_{e} / n_{0}\right)$ :

$$
\begin{aligned}
& {\left[\left(1-\delta_{e}^{2} \nabla_{\perp}^{2}\right) \frac{\partial^{4}}{\partial t^{4}}-V_{A}^{2} \nabla_{\|}^{2}\left(\left(1+\beta-\rho_{T}^{2} \nabla_{\perp}^{2}\right) \frac{\partial^{2}}{\partial t^{2}}-V_{T}^{2} \nabla_{\|}^{2}\right)+\tilde{\nu} \delta_{e}^{2} \nabla^{2} \frac{\partial^{4}}{\partial t^{4}}\right] \phi=} \\
& V_{T e}^{2}\left(\frac{\partial^{2}}{\partial t^{2}}\left[\left(1-\delta_{e}^{2} \nabla_{\perp}^{2}+\frac{m_{e}}{m_{i}}\right) N_{e}+\nabla_{\|} N_{e i}\right]+\frac{m_{e}}{m_{i}} V_{A}^{2} \nabla_{\|}^{2}\left(N_{i}-N_{e}\right)\right),
\end{aligned}
$$

where $\beta=V_{T}^{2} / V_{A}^{2}$ is due to gas compressibility, $T \equiv T_{i}+T_{e}, \delta_{s}^{2}=c^{2} m_{s} / 4 \pi n e^{2}$ is the inertial length, the subscript $\|$ denotes the direction of the equilibrium magnetic field $\mathbf{B}_{0}$ (that is $\mathbf{E}_{\|} \| \mathbf{B}_{0}$, etc.), $\Omega_{i}=e B_{0} / m_{i} c$ is the ion-cyclotron frequency, $\rho_{T}^{2}=V_{T}^{2} / \Omega_{i}^{2}$. The terms (proportional to $\beta$ ) that are due to magnetic compressibility $B_{\|}$are hidden in re-defined temperatures: $(1+\beta)^{-1} T_{s} \rightarrow T_{s}$ (thermal velocity $(1+\beta)^{-1} V_{T s}^{2} \rightarrow V_{T s}^{2}$ and so on). The nonlinear terms are given elsewhere (Voitenko \& Goossens 2000).

\section{Parametric decay of large-scale AW into small-scale AWs}

We consider the nonlinear excitation of two short length scale AWs $\left(\phi_{1}, \phi_{2}\right)$ that are in resonance with large-scale pump AW $\left(b_{\mathrm{P}}\right)$. The resonant conditions are: $\omega_{1}-\omega_{2}=\omega_{\mathrm{P}}, \mathbf{k}_{1 \perp}-\mathbf{k}_{2 \perp}=0 ; k_{1 \|}-k_{2 \|}=k_{\mathrm{P} \|}$. We search for the solution of (1) in the form of a fast oscillating wave with slowly varying amplitude, $\phi_{1,2}=\Phi_{1,2}(t) \exp \left(-i \omega_{1,2} t+i \mathbf{k}_{1,2} \cdot \mathbf{r}\right)$. We retain only the resonant nonlinear terms that are able to produce high perpendicular wavenumbers, and get the equation for the amplitude $\Phi_{1}$ :

$$
\left[\frac{\partial}{\partial t}-\gamma_{L 1}\right] \Phi_{1}=i V_{A}\left(s_{\mathrm{P}} \beta \frac{\mu_{2}\left(\mu_{2}-1\right)}{\left(\mu_{2}+1\right)^{2}}-s_{2} \frac{m_{e}}{m_{i}} \sqrt{\frac{1+\mu_{2}}{1+\delta_{e}^{2} k_{2 \perp}^{2}}}\right) \mathbf{k}_{2 \perp} \cdot \mathbf{b}_{\mathrm{P} \perp} \Phi_{2},
$$

where $s=k_{z} /\left|k_{z}\right|, \mu=\rho_{T}^{2} k_{\perp}^{2}$, and $\gamma_{L}$ is the linear decrement $\left(\gamma_{L} \approx-0.5 \nu \delta_{e}^{2} k_{\perp}^{2}\right.$ if the damping is collisional).

Using $\mathbf{k}_{1 \perp} \approx \mathbf{k}_{2 \perp} \equiv \mathbf{k}_{\perp}, s_{\mathrm{P}}=1$, and $s_{1}=-s_{2}$, we solve (2) together with a similar equation for $\Phi_{2}$. The exponentially growing solution for the wave amplitudes, $\sim \exp (\delta t)$, has indices $\delta=0.5\left(\gamma_{L 1}+\gamma_{L 2}\right) \pm 0.5 \sqrt{\left(\gamma_{L 1}-\gamma_{L 2}\right)^{2}+4 \gamma_{N L}^{2}}$, where the rate of non-linear interaction is

$$
\gamma_{N L} \approx \Omega_{i} \sqrt{\frac{\mu}{\beta}} \sqrt{\left(\frac{m_{e}}{m_{i}}\right)^{2} \frac{1+\mu}{1+\delta_{e}^{2} k_{2 \perp}^{2}}-\beta^{2} \frac{\mu^{2}(\mu-1)^{2}}{(\mu+1)^{4}}}\left|b_{\mathrm{P} \perp}\right| .
$$

\section{Nonlinear self-interaction of AWs and second harmonic genera- tion}

For $k_{\mathrm{P} \perp} \delta_{i}<1$, the equation that governs the AW amplitude $b_{\mathrm{sec}}=B_{\mathrm{sec}} / B_{0}$ ( $B_{\text {sec }}$ is the wave magnetic field), excited at the second harmonic by the pump Alfvén wave $\left(b_{\mathrm{P}}\right)$ is:

$$
\left[\frac{\partial^{2}}{\partial t^{2}}-\omega_{k}^{2}-\gamma_{c} \frac{\partial}{\partial t}\right] b_{\mathrm{sec}}=4 \beta \omega_{\mathrm{P}} k_{\mathrm{P} \perp} \delta_{i} \frac{\partial}{\partial t}\left(b_{\mathrm{P}}^{2}+c . c\right) .
$$


Here we also look for a solution of (4) in the form of a fast oscillating wave with a slowly varying amplitude: $b_{\mathrm{sec}}=\bar{b}_{\mathrm{sec}}(t) \exp \left(-i \omega_{\mathrm{sec}} t+i \mathbf{k}_{\mathrm{sec}} \cdot \mathbf{r}\right)$. If the frequency mismatch is smaller than the growth rate, $\Delta \omega\left(\equiv \omega_{\mathrm{sec}}-2 \omega_{\mathrm{P}}\right)<\gamma_{\mathrm{sec}}$, the solution to (4) is $\left|\bar{b}_{\mathrm{sec}}\right|=4 \beta \omega_{\mathrm{P}} k_{\mathrm{P} \perp} \delta_{i} \bar{b}_{\mathrm{P}}^{2} t$, and the growth rate of the process can be estimated as

$$
\gamma_{\mathrm{sec}}=4 \beta \omega_{\mathrm{P}} k_{\mathrm{P} \perp} \delta_{i} \frac{\bar{B}_{\mathrm{P}}}{B_{0}} .
$$

Both the resistive dissipation and the frequency mismatch are proportional to $k_{\perp}^{2}$ and define an upper cut-off for this process in wavenumber space. For coronal parameters, the frequency mismatch defines a more severe restriction than dissipation, and gives an upper boundary for second harmonic generation, $\left(k_{\mathrm{P} \perp} \delta_{i}\right)_{\max } \sim 0.01-0.1$, which is quite high.

\section{Discussion and Conclusions}

The parametric decay of large-scale AWs into a spectrum of short-scale AWs introduces a jump-like transport of wave energy directly into the dissipative wavenumber domain. The threshold condition, $\gamma_{N L}^{2}>\gamma_{L 1} \gamma_{L 2}$, can be satisfied in the solar corona with very small wave amplitudes $b_{\mathrm{P} \perp} \geq 10^{-5}$ (we took $\left.\Omega_{i}=10^{6}, \beta=10^{-2}\right)$. Short-scale AWs with wavenumbers $\rho_{T} k_{\perp} \sim 1$ can be excited in the corona by large-scale AWs with $b_{\mathrm{P} \perp}=5 \times 10^{-3}$ in a fraction of a second, $\tau_{N L} \approx 10^{-1} \mathrm{~s}$. This process can explain also the replenishment of small-scale AWs, observed in the solar wind (Matthaeus et al. 1999).

The process of phase-mixing, which is inevitable in non-uniform space plasmas, switches on the nonlinear excitation of Alfvén waves at the second harmonic. This process produces shorter length-scales faster than phase mixing itself, and leads also to a conversion to higher wave frequencies. The sequence of second harmonic generations results in the energy cascading towards higher perpendicular wavenumbers and frequencies. In this way, relatively high-frequency Alfvén waves may be excited by low-frequency waves at high coronal levels, where their presence is suggested by SOHO observations (Wilhelm et al. 1998; Cranmer et al. 1999, and references therein).

Acknowledgments. Research supported by the FWO-Vlaanderen grant G.0335.98

\section{References}

Cranmer S.R., Field G.B., Kohl J.L. 1999, ApJ 518, 937

Goossens M. 1994, Space Sci. Rev. 68, 51

Li X., Habbal S.R., Hollweg J.V. Esser R. 1999, J. Geophys. Res. 104, 2521

Matthaeus W. H., Zank G. P., Leamon R. J., Smith C. W., Mullan D. J. 1999, Space Sci. Rev. 87, 269

Nakariakov V. M., Roberts B., Muravski K. 1997, Solar Phys. 175, 93

Voitenko Yu. M., Goossens M. 2000, A\&A 357, 1073

Wilhelm K., Marsch E., Dwivedi B. N. et al. 1998, ApJ 500, 1023 